\title{
Editorial
}

\section{UN ENFOQUE SOBRE ALGUNOS PROBLEMAS DE LA EDUCACIÓN SUPERIOR EN LA REPÚBLICA DOMINICANA}

Había sido discutido en el seno de los organismos de gobierno de esta institución el problema que plantea para los encargados de la formación de nuevos profesionales en el país la doble vertiente que representa, por un lado, la proliferación de instituciones de Educación Superior amparadas [en] una ley que no establece reglamentos para calificarlas; y por el otro, la posibilidad de que surgieran organismos de control que pudieran impedir la ampliación de los medios de formación universitaria.

Meditábamos sobre estas ideas cuando llegó al INTEC, en el transcurso del mes de febrero el Ing. Carlos Ramos Royo, director del Instituto de Materiales y Modelos Estructurales (IMML) de la Universidad Central de Caracas y, comentando sobre estas preocupaciones, nos relató lo siguiente: el Ing. Ramos, conjuntamente con el Ing. José Graces habían sido requeridos por un grupo privado con el fin de preparar un proyecto que sería sometido al Consejo Nacional de Universidades, para la creación de una nueva Institución de Educación Superior en su país. El Consejo Nacional de Universidades es el organismo que traza las normas fundamentales para la fundación y el financiamiento de una nueva universidad, de manera que se llegue a una forma de planeamiento universitario más integral y acorde con los planos de desarrollo económico y social del país.

A fin de aceptar la creación de esa nueva universidad, sus patrocinadores debían, pues, presentar al Consejo Nacional de Universidades un mínimo de informaciones imprescindibles para su reconocimiento oficial. Entre las mismas cabe señalar las siguientes: 
1. El número de carreras que se enseñarían.

2. Programas detallados de por lo menos los tres primeros años de cada carrera.

3. Personal docente y administrativo disponible con sus respectivos curriculum vitae.

4. Plan de inversiones requeridas, tanto para facilidades físicas como para financiar el período de puesta en marcha de la Institución.

5. Una proyección de cómo seguirían desarrollándose las carreras y la incorporación de nuevas carreras al final de cada año académico.

6. Una relación del grupo promotor con sus correspondientes credenciales y capacidades directivas y administrativas.

Es este Consejo Nacional de Universidades, según nos relató el Ing. Ramos; el encargado de distribuir entre las distintas universidades la partida que en el Presupuesto Nacional está dedicada a la Educación Superior. De esa manera se armonizan las relaciones entre el poder público y las universidades ya que el Consejo Nacional de Universidades, presidido por el Ministro de Educación como representante del Estado, es un vehículo de carácter técnico para asesorar al Gobierno Nacional en el establecimiento de instituciones de educación superior y en la vigilancia de la distribución de los dineros nacionales asignados por el Estado a las universidades oficiales y privadas.

La distribución de los fondos del Estado, nos continuó diciendo el Ing. Ramos, se lleva a cabo tomando como base los proyectos de inversión que presenten los distintos Rectores, los programas de investigación que se aduzcan, los proyectos de crecimiento estudiantil y sus modalidades, todo lo cual evitará que se dupliquen innecesariamente programas, fondos y recursos humanos. Aquellos datos nos movieron a buscar nuevas fuentes de información al respecto. 
Supimos que organismos con características similares existen en otros países latinoamericanos, como es el caso de Colombia, donde se creó una Asociación Colombiana de Universidades y un Fondo Universitario Nacional, surgido a raíz de la "proliferación de universidades" en ese país. El objetivo fundamental de ese organismo es el de coordinar los esfuerzos de las diversas universidades y el de ser agencia técnica del gobierno para la distribución de los fondos públicos a las instituciones de educación superior. Dicho organismo "pretendía la solidaridad de las universidades existentes y de las nuevas que se creasen debidamente con el fin de conseguir propósito nacionales."

Nos ha parecido oportuno relatar estas experiencias traídas por el Ing. Ramos y las recogidas en otras fuentes al aparecer el primer número de nuestro órgano oficial de difusión, por considerar que tienden a enriquecer las bases que finalmente darán origen a algún esquema que lleve a unificar el sentido del crecimiento de la Educación Superior en la República Dominicana. Estas reflexiones se hacen aún más oportunas en este momento, cuando ya algunas autoridades universitarias han comenzado a expresar su preocupación por este asunto sugiriendo concretamente la creación del Consejo Nacional de Universidades.

\footnotetext{
1 Augusto Rando: “Una Experiencia Nacional: La Asociación Colombiana de Universidades. V el Planeamiento de la Educación Universitaria en Colombia". Corrientes de la Educación Superior en América. Departamento de Asuntos Educativos Unión Panamericana, Washington 1966, p. 20.
} 